\title{
Generalized harmonically convex functions on fractal sets and related Hermite-Hadamard type inequalities
}

\author{
Wenbing Sun
}

School of Science, Shaoyang University, Shaoyang 422000, Hunan, P. R. China.

Communicated by X.-J. Yang

\begin{abstract}
In this paper, the author introduced the concept of generalized harmonically convex function on fractal sets $\mathbb{R}^{\alpha}(0<\alpha \leqslant 1)$ of real line numbers and established generalized Hermite-Hadamard's inequalities for generalized harmonically convex function. Then, by creating a local fractional integral identity, obtained some Hermite-Hadamard type inequalities of these classes of functions. (C) 2017 All rights reserved.
\end{abstract}

Keywords: Generalized harmonically convex function, Hermite-Hadamard type inequality, fractal space, local fractional integral.

2010 MSC: 26D15, 26A51, 26D10.

\section{Introduction}

Let $f: I \subseteq \mathbb{R} \rightarrow \mathbb{R}$ be a convex function and $a, b \in$ I with $a<b$, then the following inequality holds,

$$
f\left(\frac{a+b}{2}\right) \leqslant \frac{1}{b-a} \int_{a}^{b} f(x) d x \leqslant \frac{f(a)+f(b)}{2}
$$

which is well-known as Hermite-Hadamard's inequality for convex functions. Both inequalities hold in the reversed direction if $f$ is concave.

With the improvement of the concept of convexity, such as s-convex, $(\alpha, m)$-convex, $(\alpha, m)$-preinvex and so on, some new results for Hermite-Hadamard's inequality were obtained. For more recent results, one can see $[2,3,5,8,11-13,15]$ and the references cited therein.

In [7], İşcan provided the definition of harmonically convexity as follows.

Definition 1.1. Let $I \subset \mathbb{R} \backslash\{0\}$ be a real interval. A function $f: I \rightarrow \mathbb{R}$ is said to be harmonically convex, if

$$
f\left(\frac{x y}{t x+(1-t) y}\right) \leqslant t f(y)+(1-t) f(x)
$$

for all $x, y \in I$ and $t \in[0,1]$. If the inequality in (1.1) is reversed, then $f$ is said to be harmonically concave.

Email address: swb0520@163.com (Wenbing Sun)

doi:10.22436/jnsa.010.11.24

Received 2017-07-07 
İşcan proved the following results in [7].

Theorem 1.2. Let $\mathrm{f}: \mathrm{I} \subset \mathbb{R} \backslash\{0\} \rightarrow \mathbb{R}$ be a harmonically convex function and $\mathrm{a}, \mathrm{b} \in \mathrm{I}$ with $\mathrm{a}<\mathrm{b}$. If $\mathrm{f} \in \mathrm{L}[\mathrm{a}, \mathrm{b}]$, then the following inequalities hold

$$
f\left(\frac{2 a b}{a+b}\right) \leqslant \frac{a b}{b-a} \int_{a}^{b} \frac{f(x)}{x^{2}} d x \leqslant \frac{f(a)+f(b)}{2} .
$$

Lemma 1.3. Let $\mathrm{f}: \mathrm{I} \subset \mathbb{R} \backslash\{0\} \rightarrow \mathbb{R}$ be a differentiable function on $\mathrm{I}^{\circ}$ ( $\mathrm{I}^{\circ}$ is the interior of $\mathrm{I}$ ) and $\mathrm{a}, \mathrm{b} \in \mathrm{I}$ with $\mathrm{a}<\mathrm{b}$. If $\mathrm{f}^{\prime} \in \mathrm{L}[\mathrm{a}, \mathrm{b}]$ then

$$
\frac{f(a)+f(b)}{2}-\frac{a b}{b-a} \int_{a}^{b} \frac{f(x)}{x^{2}} d x=\frac{a b(b-a)}{2} \int_{0}^{1} \frac{1-2 t}{(t b+(1-t) a)^{2}} f^{\prime}\left(\frac{a b}{t b+(1-t) a}\right) d t .
$$

Theorem 1.4. Let $\mathrm{f}: \mathrm{I} \subset(0, \infty) \rightarrow \mathbb{R}$ be a differentiable function on $\mathrm{I}^{\circ}, \mathrm{a}, \mathrm{b} \in \mathrm{I}$ with $\mathrm{a}<\mathrm{b}$, and $\mathrm{f}^{\prime} \in \mathrm{L}[\mathrm{a}, \mathrm{b}]$. If $\left|f^{\prime}\right|^{\mathrm{q}}$ is harmonically convex on $[\mathrm{a}, \mathrm{b}]$, then

$$
\left|\frac{f(a)+f(b)}{2}-\frac{a b}{b-a} \int_{a}^{b} \frac{f(x)}{x^{2}} d x\right| \leqslant \frac{a b(b-a)}{2} \lambda_{1}^{1-\frac{1}{q}}\left[\lambda_{2}\left|f^{\prime}(a)\right|^{q}+\lambda_{3}\left|f^{\prime}(b)\right|^{q}\right]^{\frac{1}{q}},
$$

where

$$
\begin{aligned}
& \lambda_{1}=\frac{1}{a b}-\frac{2}{(b-a)^{2}} \ln \left(\frac{(a+b)^{2}}{4 a b}\right), \\
& \lambda_{2}=\frac{-1}{b(b-a)}+\frac{3 a+b}{(b-a)^{3}} \ln \left(\frac{(a+b)^{2}}{4 a b}\right), \\
& \lambda_{3}=\frac{1}{a(b-a)}-\frac{3 b+a}{(b-a)^{3}} \ln \left(\frac{(a+b)^{2}}{4 a b}\right)=\lambda_{1}-\lambda_{2} .
\end{aligned}
$$

In recent years, the fractal theory has attracted wide attention. Since the calculus on fractal space can lead to many real world models becoming easier to understand, for instance see [21, 23], more and more researchers extended their studies to fractal space, see $[1,6,9,10,14,16]$. In [18-20, 22, 24], Yang stated the theory of local fractional calculus on fractal space systematically, and introduced some recent results on local fractional calculus.

The main aim of this paper is to introduce the concept of generalized harmonically convex function on fractal space and establish generalized Hermite-Hadamard's inequalities for generalized harmonically convex function and some other Hermite-Hadamard type inequalities involving local fractional calculus on fractal space.

\section{Preliminaries}

Let $\mathbb{R}^{\alpha}(0<\alpha \leqslant 1)$ be $\alpha$-type set of the real line numbers, and use Gao-Yang-Kang's method to describe the definitions of the local fractional derivative and local fractional integral, see [18, 19].

If $\mathrm{a}^{\alpha}, \mathrm{b}^{\alpha}, \mathrm{c}^{\alpha} \in \mathbb{R}^{\alpha}$, then

(1) $a^{\alpha}+b^{\alpha} \in \mathbb{R}^{\alpha}, a^{\alpha} b^{\alpha} \in \mathbb{R}^{\alpha}$;

(2) $a^{\alpha}+b^{\alpha}=b^{\alpha}+a^{\alpha}=(a+b)^{\alpha}=(b+a)^{\alpha}$;

(3) $a^{\alpha}+\left(b^{\alpha}+c^{\alpha}\right)=(a+b)^{\alpha}+c^{\alpha}$;

(4) $a^{\alpha} b^{\alpha}=b^{\alpha} a^{\alpha}=(a b)^{\alpha}=(b a)^{\alpha}$;

(5) $a^{\alpha}\left(b^{\alpha} c^{\alpha}\right)=\left(a^{\alpha} b^{\alpha}\right) c^{\alpha}$; 
(6) $a^{\alpha}\left(b^{\alpha}+c^{\alpha}\right)=a^{\alpha} b^{\alpha}+a^{\alpha} c^{\alpha}$;

(7) $a^{\alpha}+0^{\alpha}=0^{\alpha}+a^{\alpha}=a^{\alpha}$ and $a^{\alpha} 1^{\alpha}=1^{\alpha} a^{\alpha}=a^{\alpha}$.

Now, we state the definitions of the local fractional derivative and local fractional integral on $\mathbb{R}^{\alpha}$ as follows.

Definition 2.1 ([18]). A non-differentiable function $f: \mathbb{R} \rightarrow \mathbb{R}^{\alpha}, x \rightarrow f(x)$ is called local fractional continuous at $x_{0}$, if for any $\varepsilon>0$, there exists $\delta>0$, such that

$$
\left|f(x)-f\left(x_{0}\right)\right|<\varepsilon^{\alpha},
$$

holds for $\left|x-x_{0}\right|<\delta$, where $\varepsilon, \delta \in \mathbb{R}$. If $f(x)$ is local fractional continuous on $(a, b)$, we denote $f(x) \in$ $\mathrm{C}_{\alpha}(\mathrm{a}, \mathrm{b})$.

Definition 2.2 ([18]). The local fractional derivative of $f(x)$ of order $\alpha$ at $x=x_{0}$ is defined by

$$
f^{(\alpha)}\left(x_{0}\right)=\left.\frac{d^{\alpha} f(x)}{d x^{\alpha}}\right|_{x=x_{0}}=\lim _{x \rightarrow x_{0}} \frac{\Gamma(\alpha+1)\left(f(x)-f\left(x_{0}\right)\right)}{\left(x-x_{0}\right)^{\alpha}} .
$$

$D_{\alpha}(a, b)$ is called $\alpha$-local fractional derivative set. If there exists $f^{((k+1) \alpha)}(x)=\overbrace{D_{x}^{\alpha} \cdots D_{x}^{\alpha}}^{(n+1) \text { times }} f(x)$ for any $x \in I \subseteq \mathbb{R}$, then we denote $f \in D_{(n+1) \alpha}(I)$, where $n=0,1,2, \cdots$.

Definition 2.3 ([18]). Let $f(x) \in C_{\alpha}[a, b]$. The local fractional integral of function $f(x)$ of order $\alpha$ is defined by

$$
{ }_{a} I_{b}^{(\alpha)} f(x)=\frac{1}{\Gamma(\alpha+1)} \int_{a}^{b} f(t)(d t)^{\alpha}=\frac{1}{\Gamma(\alpha+1)} \lim _{\Delta t \rightarrow 0} \sum_{j=0}^{N-1} f\left(t_{j}\right)\left(\Delta t_{j}\right)^{\alpha},
$$

where $a=t_{0}<t_{1}<\cdots<t_{N-1}<t_{N}=b,\left[t_{j}, t_{j+1}\right]$ is a partition of the interval $[a, b], \Delta t_{j}=t_{j+1}-t_{j}, \Delta t=$ $\max \left\{\Delta \mathrm{t}_{0}, \Delta \mathrm{t}_{1}, \cdots, \Delta \mathrm{t}_{\mathrm{N}-1}\right\}$.

Note that ${ }_{a} I_{a}^{(\alpha)} f(x)=0$, and ${ }_{a} I_{b}^{(\alpha)} f(x)=-{ }_{b} I_{a}^{(\alpha)} f(x)$ if $a<b$. We denote $f(x) \in I_{x}^{(\alpha)}[a, b]$, if there exists ${ }_{a} I_{x}^{\alpha} f(x)$ for any $x \in[a, b]$.

Definition 2.4 ([10]). Let $f: I \subseteq \mathbb{R} \rightarrow \mathbb{R}^{\alpha}$. For any $x_{1}, x_{2} \in \mathrm{I}$ and $\lambda \in[0,1]$, if the following inequality

$$
f\left(\lambda x_{1}+(1-\lambda) x_{2}\right) \leqslant \lambda^{\alpha} f\left(x_{1}\right)+(1-\lambda)^{\alpha} f\left(x_{2}\right),
$$

holds, then $f$ is called a generalized convex function on I.

Lemma 2.5 ([18]).

(1) Suppose that $f(x)=g^{(\alpha)}(x) \in C_{\alpha}[a, b]$, then

$$
{ }_{a} I_{b}^{(\alpha)} f(x)=g(b)-g(a) .
$$

(2) Suppose that $f(x), g(x) \in D_{\alpha}[a, b]$, and $f^{(\alpha)}(x), g^{(\alpha)}(x) \in C_{\alpha}[a, b]$, then

$$
{ }_{a} I_{b}^{(\alpha)} f(x) g^{(\alpha)}(x)=\left.f(x) g(x)\right|_{a} ^{b}-{ }_{a} I_{b}^{(\alpha)} f^{(\alpha)}(x) g(x) \text {. }
$$

Lemma 2.6 ([18]).

$$
\begin{aligned}
\frac{\mathrm{d}^{\alpha} x^{k \alpha}}{d x^{\alpha}} & =\frac{\Gamma(1+k \alpha)}{\Gamma(1+(k-1) \alpha)} x^{(k-1) \alpha} \\
\frac{1}{\Gamma(\alpha+1)} \int_{a}^{b} x^{k \alpha}(d x)^{\alpha} & =\frac{\Gamma(1+k \alpha)}{\Gamma(1+(k+1) \alpha)}\left(b^{(k+1) \alpha}-a^{(k+1) \alpha}\right), \quad k>0 .
\end{aligned}
$$


Lemma 2.7 ([4, 17, Generalized Hölder's inequality]). Let $f, g \in C_{\alpha}[a, b], p, q>1$, with $\frac{1}{p}+\frac{1}{q}=1$, then

$$
\frac{1}{\Gamma(\alpha+1)} \int_{a}^{b}|f(x) g(x)|(d x)^{\alpha} \leqslant\left(\frac{1}{\Gamma(\alpha+1)} \int_{a}^{b}|f(x)|^{p}(d x)^{\alpha}\right)^{1 / p}\left(\frac{1}{\Gamma(\alpha+1)} \int_{a}^{b}|g(x)|^{q}(d x)^{\alpha}\right)^{1 / q}
$$

Lemma 2.8 ([18]).

$$
{ }_{\mathrm{a}} \mathrm{I}_{\mathrm{b}}^{(\alpha)} 1=\frac{(\mathrm{b}-\mathrm{a})^{\alpha}}{\Gamma(1+\alpha)}
$$

\section{Generalized harmonically convex function}

Now, we provide the definition of generalized harmonically convex function on fractal space as follows.

Definition 3.1. Let $I \subset \mathbb{R} \backslash\{0\}$ be a real interval. A function $f: I \rightarrow \mathbb{R}^{\alpha}(0<\alpha \leqslant 1)$ is said to be generalized harmonically convex, if

$$
f\left(\frac{x y}{t x+(1-t) y}\right) \leqslant t^{\alpha} f(y)+(1-t)^{\alpha} f(x)
$$

for all $x, y \in I$ and $t \in[0,1]$. If the inequality in (3.1) is reversed, then $f$ is said to be generalized harmonically concave.

Here are two examples of this kind of functions.

Example 3.2. Let $f:(0, \infty) \rightarrow \mathbb{R}^{\alpha}$ and $g:(-\infty, 0) \rightarrow \mathbb{R}^{\alpha}$, then $f(x)=x^{\alpha}$ is a generalized harmonically convex function and $g(x)=x^{\alpha}$ is a generalized harmonically concave function.

Proof. Let $x_{1}, x_{2} \in(0, \infty)$ and $t \in[0,1]$. By simple calculating, we have

$$
\begin{aligned}
{\left[\mathrm{t}^{\alpha} x_{2}^{\alpha}+(1-\mathrm{t})^{\alpha} x_{1}^{\alpha}\right]\left[\mathrm{t}^{\alpha} x_{1}^{\alpha}+(1-\mathrm{t})^{\alpha} x_{2}^{\alpha}\right] } & =\left[\mathrm{t}^{2 \alpha}+(1-\mathrm{t})^{2 \alpha}\right] x_{1}^{\alpha} x_{2}^{\alpha}+\left(x_{1}^{2 \alpha}+x_{2}^{2 \alpha}\right) t^{\alpha}(1-t)^{\alpha} \\
& \geqslant\left[t^{2 \alpha}+(1-t)^{2 \alpha}\right] x_{1}^{\alpha} x_{2}^{\alpha}+2 t^{\alpha}(1-t)^{\alpha} x_{1}^{\alpha} x_{2}^{\alpha} \\
& =\left[t^{\alpha}+(1-t)^{\alpha}\right]^{2} x_{1}^{\alpha} x_{2}^{\alpha} \\
& =x_{1}^{\alpha} x_{2}^{\alpha} .
\end{aligned}
$$

Since $f(x)=x^{\alpha}$, we further have

$$
f\left(\frac{x_{1} x_{2}}{t x_{1}+(1-t) x_{2}}\right)=\frac{x_{1}^{\alpha} x_{2}^{\alpha}}{t^{\alpha} x_{1}^{\alpha}+(1-t)^{\alpha} x_{2}^{\alpha}} \leqslant t^{\alpha} x_{2}^{\alpha}+(1-t)^{\alpha} x_{1}^{\alpha}=t^{\alpha} f\left(x_{2}\right)+(1-t)^{\alpha} f\left(x_{1}\right) .
$$

Thus, $f(x)=x^{\alpha}$ is a generalized harmonically convex function. From similar method, it is easy to prove that $g(x)=x^{\alpha}$ is a generalized harmonically concave function.

Some properties of the generalized harmonically convex functions will be studied as follows.

Proposition 3.3. If $f:(0, \infty) \rightarrow \mathbb{R}^{\alpha}$ is generalized convex (Definition 2.4) and nondecreasing, then $f$ is generalized harmonically convex.

Proof. Let $x, y \in(0, \infty)$ and $t \in[0,1]$. According to the method of Example 3.2, it is easy to prove that

$$
0<\frac{x y}{t x+(1-t) y} \leqslant t y+(1-t) x
$$

Since $f$ is nondecreasing and generalized convex on $(0, \infty)$, we have

$$
f\left(\frac{x y}{t x+(1-t) y}\right) \leqslant f(t y+(1-t) x) \leqslant t^{\alpha} f(y)+(1-t)^{\alpha} f(x)
$$

Hence, $f$ is generalized harmonically convex. 
Proposition 3.4. If $\mathrm{f}:(0, \infty) \rightarrow \mathbb{R}^{\alpha}$ is generalized harmonically convex and nonincreasing, then $\mathrm{f}$ is generalized convex.

Proof. Let $x, y \in(0, \infty)$ and $t \in[0,1]$. It is easy to prove that

$$
0<\frac{x y}{t x+(1-t) y} \leqslant t y+(1-t) x
$$

Since $f$ is nonincreasing and generalized harmonically convex on $(0, \infty)$, we have

$$
t^{\alpha} f(y)+(1-t)^{\alpha} f(x) \geqslant f\left(\frac{x y}{t x+(1-t) y}\right) \geqslant f(t y+(1-t) x)
$$

From Definition 2.4, $\mathrm{f}$ is generalized convex.

Similarly, we can obtain the following two propositions.

Proposition 3.5. If $\mathrm{f}:(-\infty, 0) \rightarrow \mathbb{R}^{\alpha}$ is generalized convex and nonincreasing, then $\mathrm{f}$ is generalized harmonically convex.

Proposition 3.6. If $\mathrm{f}:(-\infty, 0) \rightarrow \mathbb{R}^{\alpha}$ is generalized harmonically convex and nondecreasing, then $\mathrm{f}$ is generalized convex.

\section{Some results related Hermite-Hadamard type inequalities}

Hermite-Hadamard's inequalities for generalized harmonically convex on fractal space can be represented as follows.

Theorem 4.1. Let $\mathrm{f}: \mathrm{I} \subset \mathbb{R} \backslash\{0\} \rightarrow \mathbb{R}^{\alpha}$ be a generalized harmonically convex function on fractal space and $\mathrm{a}, \mathrm{b} \in \mathrm{I}$ with $\mathrm{a}<\mathrm{b}$. If $\mathrm{f}(\mathrm{x}) \in \mathrm{I}_{x}^{(\alpha)}[\mathrm{a}, \mathrm{b}]$, then

$$
\frac{1}{\Gamma(1+\alpha)} f\left(\frac{2 a b}{a+b}\right) \leqslant \frac{a^{\alpha} b^{\alpha}}{(b-a)^{\alpha}} a I_{b}^{(\alpha)} \frac{f(x)}{x^{2 \alpha}} \leqslant \frac{\Gamma(1+\alpha)}{\Gamma(1+2 \alpha)}[f(a)+f(b)] .
$$

Proof. Since $f$ is a generalized harmonically convex function on $[a, b]$, setting $t=\frac{1}{2}$ in the inequality (3.1), we have for all $x, y \in[a, b]$

$$
f\left(\frac{2 x y}{x+y}\right) \leqslant \frac{f(x)+f(y)}{2^{\alpha}}
$$

Choosing $x=\frac{a b}{t b+(1-t) a}, y=\frac{a b}{t a+(1-t) b}$, we obtain

$$
f\left(\frac{2 a b}{a+b}\right) \leqslant \frac{f\left(\frac{a b}{t b+(1-t) a}\right)+f\left(\frac{a b}{t a+(1-t) b}\right)}{2^{\alpha}} .
$$

Integrating the above inequality with respect to $t$ over $[0,1]$, we obtain

$$
\begin{aligned}
\frac{1}{\Gamma(1+\alpha)} f\left(\frac{2 a b}{a+b}\right) & \leqslant \frac{1}{2^{\alpha}}\left[\frac{1}{\Gamma(1+\alpha)} \int_{0}^{1} f\left(\frac{a b}{t b+(1-t) a}\right)(d t)^{\alpha}+\frac{1}{\Gamma(1+\alpha)} \int_{0}^{1} f\left(\frac{a b}{t a+(1-t) b}\right)(d t)^{\alpha}\right] \\
& =\frac{1}{2^{\alpha}}\left(\frac{a b}{b-a}\right)^{\alpha}\left[\frac{1}{\Gamma(1+\alpha)} \int_{a}^{b} \frac{f(x)}{x^{2 \alpha}}(d x)^{\alpha}+\frac{1}{\Gamma(1+\alpha)} \int_{a}^{b} \frac{f(y)}{y^{2 \alpha}}(d y)^{\alpha}\right] \\
& =\left(\frac{a b}{b-a}\right)^{\alpha}{ }_{a} I_{b}^{(\alpha)} \frac{f(x)}{x^{2 \alpha}}
\end{aligned}
$$


where we have used the fact that

$$
\frac{1}{\Gamma(1+\alpha)} \int_{0}^{1} f\left(\frac{2 a b}{a+b}\right)(d t)^{\alpha}=\frac{1}{\Gamma(1+\alpha)} f\left(\frac{2 a b}{a+b}\right) .
$$

For the proof of the second inequality in (4.1), we note that $f$ is a generalized harmonically convex function, for $t \in[0,1]$, we have

$$
f\left(\frac{a b}{t b+(1-t) a}\right) \leqslant t^{\alpha} f(a)+(1-t)^{\alpha} f(b)
$$

and

$$
f\left(\frac{a b}{t a+(1-t) b}\right) \leqslant t^{\alpha} f(b)+(1-t)^{\alpha} f(a) .
$$

Adding the above two inequalities, we get

$$
f\left(\frac{a b}{t b+(1-t) a}\right)+\left(\frac{a b}{t a+(1-t) b}\right) \leqslant t^{\alpha}[f(a)+f(b)]+(1-t)^{\alpha}[f(b)+f(a)]
$$

Integrating the above inequality with respect to $t$ over $[0,1]$, we have

$$
\frac{a^{\alpha} b^{\alpha}}{(b-a)^{\alpha}} a I_{b}^{(\alpha)} \frac{f(x)}{x^{2 \alpha}} \leqslant \frac{\Gamma(1+\alpha)}{\Gamma(1+2 \alpha)}[f(a)+f(b)]
$$

where we have used the fact that

$$
\frac{1}{\Gamma(1+\alpha)} \int_{0}^{1}(1-t)^{\alpha}(d t)^{\alpha}=\frac{1}{\Gamma(1+\alpha)} \int_{0}^{1} t^{\alpha}(d t)^{\alpha}=\frac{\Gamma(1+\alpha)}{\Gamma(1+2 \alpha)} .
$$

The proof is completed.

Remark 4.2. In Theorem 4.1, we take $\alpha=1$, then inequalities (4.1) reduces to inequalities (1.2).

Lemma 4.3. Let $\mathrm{I} \subset \mathbb{R} \backslash\{0\}$ be an interval, $f: \mathrm{I}^{\circ} \subset \mathbb{R} \backslash\{0\} \rightarrow \mathbb{R}^{\alpha}$ ( $\mathrm{I}^{\circ}$ is the interior of $\mathrm{I}$ ) such that $\mathrm{f} \in \mathrm{D}_{\alpha}\left(\mathrm{I}^{\circ}\right)$ and $\mathrm{f}^{(\alpha)} \in \mathrm{C}_{\alpha}(\mathrm{a}, \mathrm{b})$ for $\mathrm{a}, \mathrm{b} \in \mathrm{I}^{\circ}$ with $\mathrm{a}<\mathrm{b}$. Then the following equality holds.

$$
\begin{aligned}
\frac{f(a)+f(b)}{2^{\alpha}} & -\Gamma(1+\alpha) \frac{a^{\alpha} b^{\alpha}}{(b-a)^{\alpha}} \mathrm{I}_{b}^{(\alpha)} \frac{f(x)}{x^{2 \alpha}} \\
& =\frac{a^{\alpha} b^{\alpha}(b-a)^{\alpha}}{2^{\alpha}} \frac{1}{\Gamma(1+\alpha)} \int_{0}^{1} \frac{(1-2 t)^{\alpha}}{(t b+(1-t) a)^{2 \alpha}} f^{(\alpha)}\left(\frac{a b}{t b+(1-t) a}\right)(d t)^{\alpha} .
\end{aligned}
$$

Proof. Let

$$
I_{r}=\frac{a^{\alpha} b^{\alpha}(b-a)^{\alpha}}{2^{\alpha}} \frac{1}{\Gamma(1+\alpha)} \int_{0}^{1} \frac{(1-2 t)^{\alpha}}{(t b+(1-t) a)^{2 \alpha}} f^{(\alpha)}\left(\frac{a b}{t b+(1-t) a}\right)(d t)^{\alpha} .
$$

By the local fractional integration by parts, we have

$$
\begin{aligned}
I_{r} & =\left.\frac{(2 t-1)^{\alpha}}{2^{\alpha}} f\left(\frac{a b}{t b+(1-t) a}\right)\right|_{0} ^{1}-\frac{1}{\Gamma(1+\alpha)} \int_{0}^{1} \Gamma(1+\alpha) f\left(\frac{a b}{t b+(1-t) a}\right)(d t)^{\alpha} \\
& =\frac{f(a)+f(b)}{2^{\alpha}}-\frac{\Gamma(1+\alpha)}{\Gamma(1+\alpha)} \int_{0}^{1} f\left(\frac{a b}{t b+(1-t) a}\right)(d t)^{\alpha} .
\end{aligned}
$$

Using changing variable with $x=\frac{a b}{t b+(1-t) a}$, we obtain

$$
\begin{aligned}
I_{r} & =\frac{f(a)+f(b)}{2^{\alpha}}-\Gamma(1+\alpha)\left(\frac{a b}{b-a}\right)^{\alpha} \frac{1}{\Gamma(1+\alpha)} \int_{a}^{b} \frac{f(x)}{x^{2 \alpha}}(d x)^{\alpha} \\
& =\frac{f(a)+f(b)}{2^{\alpha}}-\Gamma(1+\alpha) \frac{a^{\alpha} b^{\alpha}}{(b-a)^{\alpha}} I_{b}^{(\alpha)} \frac{f(x)}{x^{2 \alpha}} .
\end{aligned}
$$


This completes the proof.

Remark 4.4. In Lemma 4.3, we take $\alpha=1$, then equality (4.2) reduces to equality (1.3).

Theorem 4.5. Let $\mathrm{I} \subset(0, \infty)$ be an interval, $\mathrm{f}: \mathrm{I}^{\circ} \rightarrow \mathbb{R}^{\alpha}$ ( $\mathrm{I}^{\circ}$ is the interior of $\mathrm{I}$ ) such that $\mathrm{f} \in \mathrm{D}_{\alpha}\left(\mathrm{I}^{\circ}\right)$ and $\mathrm{f}^{(\alpha)} \in \mathrm{C}_{\alpha}[\mathrm{a}, \mathrm{b}]$ for $\mathrm{a}, \mathrm{b} \in \mathrm{I}^{\circ}$ with $\mathrm{a}<\mathrm{b}$. If $\left|\mathrm{f}^{(\alpha)}\right|^{\mathrm{q}}$ is generalized harmonically convex on $[\mathrm{a}, \mathrm{b}]$ for $\mathrm{q}>1$, then for all $x \in[a, b]$, the following inequality holds.

$$
\begin{aligned}
\left|\frac{f(a)+f(b)}{2^{\alpha}}-\Gamma(1+\alpha) \frac{a^{\alpha} b^{\alpha}}{(b-a)^{\alpha}} a I_{b}^{(\alpha)} \frac{f(x)}{x^{2 \alpha}}\right| \leqslant & \frac{a^{\alpha} b^{\alpha}(b-a)^{\alpha}}{2^{\alpha}}\left(\lambda_{1}^{\alpha}\right)^{1-\frac{1}{q}} \\
& \times\left[\lambda_{2}^{\alpha}\left|f^{(\alpha)}(a)\right|^{q}+\lambda_{3}^{\alpha}\left|f^{(\alpha)}(b)\right|^{q}\right]^{\frac{1}{q}},
\end{aligned}
$$

where

$$
\begin{aligned}
& \lambda_{1}^{\alpha}=\frac{1}{a^{\alpha} b^{\alpha} \Gamma(1+\alpha)}+\frac{2^{\alpha}}{(b-a)^{2 \alpha}}\left(\ln _{\alpha}\left(a^{\alpha}\right)+\ln _{\alpha}\left(b^{\alpha}\right)-2^{\alpha} \ln _{\alpha}\left(\frac{a+b}{2}\right)^{\alpha}\right), \\
& \lambda_{2}^{\alpha}=-\frac{1}{b^{\alpha}(b-a)^{\alpha} \Gamma(1+\alpha)}+\frac{(b+3 a)^{\alpha}}{(b-a)^{3 \alpha}}\left(2^{\alpha} \ln _{\alpha}\left(\frac{a+b}{2}\right)^{\alpha}-\ln _{\alpha}\left(a^{\alpha}\right)-\ln _{\alpha}\left(b^{\alpha}\right)\right), \\
& \lambda_{3}^{\alpha}=\frac{1}{a^{\alpha}(b-a)^{\alpha} \Gamma(1+\alpha)}-\frac{(3 b+a)^{\alpha}}{(b-a)^{3 \alpha}}\left(2^{\alpha} \ln _{\alpha}\left(\frac{a+b}{2}\right)^{\alpha}-\ln _{\alpha}\left(a^{\alpha}\right)-\ln _{\alpha}\left(b^{\alpha}\right)\right),
\end{aligned}
$$

and $\ln _{\alpha}\left(x^{\alpha}\right)$ denotes the inverse function of the Mittag-Leffler function $\mathrm{E}_{\alpha}\left(\chi^{\alpha}\right)=\sum_{\mathrm{k}=0}^{\infty} \frac{\chi^{\mathrm{k} \alpha}}{\Gamma(1+\mathrm{k} \alpha)}$ (see [18]) on fractal set.

Proof. Taking modulus in Lemma 4.3 and using the generalized Hölder's inequality (Lemma 2.7), we have

$$
\begin{aligned}
\mid \frac{f(a)+f(b)}{2^{\alpha}} & -\Gamma(1+\alpha) \frac{a^{\alpha} b^{\alpha}}{(b-a)^{\alpha}} \mathrm{I}_{b}^{(\alpha)} \frac{f(x)}{x^{2 \alpha}} \mid \\
\leqslant & \frac{a^{\alpha} b^{\alpha}(b-a)^{\alpha}}{2^{\alpha}} \frac{1}{\Gamma(1+\alpha)} \int_{0}^{1}\left|\frac{(1-2 t)^{\alpha}}{(t b+(1-t) a)^{2 \alpha}}\right|\left|f^{(\alpha)}\left(\frac{a b}{t b+(1-t) a}\right)\right|(d t)^{\alpha} \\
\leqslant & \left.\frac{a^{\alpha} b^{\alpha}(b-a)^{\alpha}}{2^{\alpha}}\left|\frac{1}{\Gamma(1+\alpha)} \int_{0}^{1}\right| \frac{(1-2 t)^{\alpha}}{(t b+(1-t) a)^{2 \alpha}} \mid(d t)^{\alpha}\right]^{1-\frac{1}{q}} \\
& \times\left[\frac{1}{\Gamma(1+\alpha)} \int_{0}^{1}\left|\frac{(1-2 t)^{\alpha}}{(t b+(1-t) a)^{2 \alpha}} \| f^{(\alpha)}\left(\frac{a b}{t b+(1-t) a}\right)\right|^{q}(d t)^{\alpha}\right]^{\frac{1}{q}} .
\end{aligned}
$$

Since $\left|f^{(\alpha)}\right|^{q}$ is generalized harmonically convex on $[a, b]$, thus

$$
\begin{aligned}
& \left.\frac{1}{\Gamma(1+\alpha)} \int_{0}^{1}\left|\frac{(1-2 t)^{\alpha}}{(t b+(1-t) a)^{2 \alpha}}\right| f^{(\alpha)}\left(\frac{a b}{t b+(1-t) a}\right)\right|^{q}(d t)^{\alpha} \\
& \leqslant \frac{1}{\Gamma(1+\alpha)} \int_{0}^{1}\left|\frac{(1-2 t)^{\alpha}}{(t b+(1-t) a)^{2 \alpha}}\right|\left(t^{\alpha}\left|f^{(\alpha)}(a)\right|^{q}+(1-t)^{\alpha}\left|f^{(\alpha)}(b)\right|^{q}\right)(d t)^{\alpha} \\
& \quad=\left(\frac{1}{\Gamma(1+\alpha)} \int_{0}^{1} \frac{|1-2 t|^{\alpha} t^{\alpha}}{(t b+(1-t) a)^{2 \alpha}}(d t)^{\alpha}\right)\left|f^{(\alpha)}(a)\right|^{q} \\
& \quad+\left(\frac{1}{\Gamma(1+\alpha)} \int_{0}^{1} \frac{|1-2 t|^{\alpha}(1-t)^{\alpha}}{(t b+(1-t) a)^{2 \alpha}}(d t)^{\alpha}\right)\left|f^{(\alpha)}(b)\right|^{q} .
\end{aligned}
$$


Applying the change of the variable $t b+(1-t) a=x$, we have

$$
\begin{aligned}
& \frac{1}{\Gamma(1+\alpha)} \int_{0}^{1} \frac{|1-2 t|^{\alpha}}{(t b+(1-t) a)^{2 \alpha}}(d t)^{\alpha} \\
&=\frac{1}{\Gamma(1+\alpha)} \int_{0}^{\frac{1}{2}} \frac{(1-2 t)^{\alpha}}{(t b+(1-t) a)^{2 \alpha}}(d t)^{\alpha}+\frac{1}{\Gamma(1+\alpha)} \int_{\frac{1}{2}}^{1} \frac{(2 t-1)^{\alpha}}{(t b+(1-t) a)^{2 \alpha}}(d t)^{\alpha} \\
&= \frac{1}{(b-a)^{2 \alpha}}\left[\frac{1}{\Gamma(1+\alpha)} \int_{a}^{\frac{a+b}{2}}\left(\frac{(a+b)^{\alpha}}{x^{2 \alpha}}-\frac{2^{\alpha}}{x^{\alpha}}\right)(d x)^{\alpha}\right. \\
&\left.+\frac{1}{\Gamma(1+\alpha)} \int_{\frac{a+b}{2}}^{b}\left(\frac{2^{\alpha}}{x^{\alpha}}-\frac{(a+b)^{\alpha}}{x^{2 \alpha}}\right)(d x)^{\alpha}\right] \\
&= \frac{1}{(b-a)^{2 \alpha}}\left[\frac{1}{\Gamma(1+\alpha)} \int_{a}^{\frac{a+b}{2}} \frac{(a+b)^{\alpha}}{x^{2 \alpha}}(d x)^{\alpha}-\frac{1}{\Gamma(1+\alpha)} \int_{a}^{\frac{a+b}{2}} \frac{2^{\alpha}}{x^{\alpha}}(d x)^{\alpha}\right. \\
&\left.+\frac{1}{\Gamma(1+\alpha)} \int_{\frac{a+b}{2}}^{b} \frac{2^{\alpha}}{x^{\alpha}}(d x)^{\alpha}-\frac{1}{\Gamma(1+\alpha)} \int_{\frac{a+b}{2}}^{b} \frac{(a+b)^{\alpha}}{x^{2 \alpha}}(d x)^{\alpha}\right]
\end{aligned}
$$

Applying the change of the variable $\frac{1}{x}=u$, and $\frac{1}{x^{2 \alpha}}(d x)^{\alpha}=-(d u)^{\alpha}$, from Lemma 2.8 , we obtain

$$
\frac{1}{\Gamma(1+\alpha)} \int_{a}^{\frac{a+b}{2}} \frac{1}{x^{2 \alpha}}(d x)^{\alpha}=-\frac{1}{\Gamma(1+\alpha)} \int_{\frac{1}{a}}^{\frac{2}{a+b}}(d u)^{\alpha}=\frac{1}{\Gamma(1+\alpha)}\left(\frac{1}{a}-\frac{2}{a+b}\right)^{\alpha} .
$$

Similarly,

$$
\frac{1}{\Gamma(1+\alpha)} \int_{\frac{a+b}{2}}^{b} \frac{1}{x^{2 \alpha}}(d x)^{\alpha}=\frac{1}{\Gamma(1+\alpha)}\left(\frac{2}{a+b}-\frac{1}{b}\right)^{\alpha}
$$

By the facts that

$$
\frac{1}{\Gamma(1+\alpha)} \int_{a}^{\frac{a+b}{2}} \frac{1}{x^{\alpha}}(d x)^{\alpha}=\ln _{\alpha}\left(\frac{a+b}{2}\right)^{\alpha}-\ln _{\alpha}\left(a^{\alpha}\right)
$$

and

$$
\frac{1}{\Gamma(1+\alpha)} \int_{\frac{a+b}{2}}^{b} \frac{1}{x^{\alpha}}(d x)^{\alpha}=\ln _{\alpha}\left(b^{\alpha}\right)-\ln _{\alpha}\left(\frac{a+b}{2}\right)^{\alpha},
$$

where $\ln _{\alpha}\left(x^{\alpha}\right)$ denotes the inverse function of the Mittag-Leffler function on fractal set.

Substituting (4.7), (4.8), (4.9), (4.10) into (4.6), we have

$$
\begin{aligned}
\lambda_{1}^{\alpha}= & \frac{1}{\Gamma(1+\alpha)} \int_{0}^{1} \frac{|1-2 t|^{\alpha}}{(t b+(1-t) a)^{2 \alpha}}(d t)^{\alpha} \\
= & \frac{1}{(b-a)^{2 \alpha} \Gamma(1+\alpha)}\left[(a+b)^{\alpha}\left(\left(\frac{1}{a}-\frac{2}{a+b}\right)^{\alpha}-\left(\frac{2}{a+b}-\frac{1}{b}\right)^{\alpha}\right)\right. \\
& \left.+2^{\alpha} \Gamma(1+\alpha)\left(\ln _{\alpha}\left(a^{\alpha}\right)+\ln _{\alpha}\left(b^{\alpha}\right)-2^{\alpha} \ln _{\alpha}\left(\frac{a+b}{2}\right)^{\alpha}\right)\right] \\
= & \frac{1}{a^{\alpha} b^{\alpha} \Gamma(1+\alpha)}+\frac{2^{\alpha}}{(b-a)^{2 \alpha}}\left(\ln _{\alpha}\left(a^{\alpha}\right)+\ln _{\alpha}\left(b^{\alpha}\right)-2^{\alpha} \ln _{\alpha}\left(\frac{a+b}{2}\right)^{\alpha}\right) .
\end{aligned}
$$


Using the same method, we can check that

$$
\begin{aligned}
\lambda_{2}^{\alpha} & =\frac{1}{\Gamma(1+\alpha)} \int_{0}^{1} \frac{|1-2 t|^{\alpha} t^{\alpha}}{(t b+(1-t) a)^{2 \alpha}}(d t)^{\alpha} \\
& =-\frac{1}{b^{\alpha}(b-a)^{\alpha} \Gamma(1+\alpha)}+\frac{(b+3 a)^{\alpha}}{(b-a)^{3 \alpha}}\left(2^{\alpha} \ln _{\alpha}\left(\frac{a+b}{2}\right)^{\alpha}-\ln _{\alpha}\left(a^{\alpha}\right)-\ln _{\alpha}\left(b^{\alpha}\right)\right),
\end{aligned}
$$

and

$$
\begin{aligned}
\lambda_{3}^{\alpha} & =\frac{1}{\Gamma(1+\alpha)} \int_{0}^{1} \frac{|1-2 t|^{\alpha}(1-t)^{\alpha}}{(t b+(1-t) a)^{2 \alpha}}(d t)^{\alpha} \\
& =\frac{1}{a^{\alpha}(b-a)^{\alpha} \Gamma(1+\alpha)}-\frac{(3 b+a)^{\alpha}}{(b-a)^{3 \alpha}}\left(2^{\alpha} \ln _{\alpha}\left(\frac{a+b}{2}\right)^{\alpha}-\ln _{\alpha}\left(a^{\alpha}\right)-\ln _{\alpha}\left(b^{\alpha}\right)\right) .
\end{aligned}
$$

Using (4.4)-(4.5) and (4.11), (4.12), (4.13), we get (4.3) which completes the proof.

Remark 4.6. In Theorem 4.5, we take $\alpha=1$, then inequality (4.3) reduces to inequality (1.4).

Theorem 4.7. Let $\mathrm{I} \subset(0, \infty)$ be an interval, $\mathrm{f}: \mathrm{I}^{\circ} \rightarrow \mathbb{R}^{\alpha}$ such that $\mathrm{f} \in \mathrm{D}_{\alpha}\left(\mathrm{I}^{\circ}\right)$ and $\mathrm{f}^{(\alpha)} \in \mathrm{C}_{\alpha}[\mathrm{a}, \mathrm{b}]$ for $\mathrm{a}, \mathrm{b} \in \mathrm{I}^{\circ}$ with $\mathrm{a}<\mathrm{b}$. If $\left|\mathrm{f}^{(\alpha)}\right|^{\mathrm{q}}$ is generalized harmonically convex on $[\mathrm{a}, \mathrm{b}]$ for $\mathrm{q}>1, \frac{1}{\mathrm{p}}+\frac{1}{\mathrm{q}}=1$, then for all $\mathrm{x} \in[\mathrm{a}, \mathrm{b}]$, the following inequality holds.

$$
\begin{aligned}
& \left|\frac{f(a)+f(b)}{2^{\alpha}}-\Gamma(1+\alpha) \frac{a^{\alpha} b^{\alpha}}{(b-a)^{\alpha}} a_{b}^{(\alpha)} \frac{f(x)}{x^{2 \alpha}}\right| \\
& \leqslant \frac{a^{\alpha} b^{\alpha}(b-a)^{\alpha}}{2^{\alpha}}\left[\frac{\Gamma(1+p \alpha)}{\Gamma(1+(p+1) \alpha)}\right]^{\frac{1}{p}}\left[\mu_{1}^{\alpha}\left|f^{(\alpha)}(a)\right|^{q}+\mu_{2}^{\alpha}\left|f^{(\alpha)}(b)\right|^{q}\right]^{\frac{1}{q}},
\end{aligned}
$$

where

$$
\begin{aligned}
& \mu_{1}^{\alpha}=\frac{1}{(b-a)^{2 \alpha} \Gamma(1+\alpha)}\left[\frac{\left(b^{2-2 q}-a^{2-2 q}\right)^{\alpha}}{(2-2 q)^{\alpha}}-\frac{a^{\alpha}\left(b^{1-2 q}-a^{1-2 q}\right)^{\alpha}}{(1-2 q)^{\alpha}}\right], \\
& \mu_{2}^{\alpha}=\frac{1}{(b-a)^{2 \alpha} \Gamma(1+\alpha)}\left[\frac{b^{\alpha}\left(b^{1-2 q}-a^{1-2 q}\right)^{\alpha}}{(1-2 q)^{\alpha}}-\frac{\left(b^{2-2 q}-a^{2-2 q}\right)^{\alpha}}{(2-2 q)^{\alpha}}\right] .
\end{aligned}
$$

Proof. Taking modulus in Lemma 4.3 and using the generalized Hölder's inequality, we have

$$
\begin{aligned}
& \left|\frac{f(a)+f(b)}{2^{\alpha}}-\Gamma(1+\alpha) \frac{a^{\alpha} b^{\alpha}}{(b-a)^{\alpha}} a_{b} I_{b}^{(\alpha)} \frac{f(x)}{x^{2 \alpha}}\right| \\
& \leqslant \frac{a^{\alpha} b^{\alpha}(b-a)^{\alpha}}{2^{\alpha}} \frac{1}{\Gamma(1+\alpha)} \int_{0}^{1}|1-2 t|^{\alpha}\left|\frac{1}{(t b+(1-t) a)^{2 \alpha}} f^{(\alpha)}\left(\frac{a b}{t b+(1-t) a}\right)\right|(d t)^{\alpha} \\
& \leqslant \\
& \quad \times\left[\frac{a^{\alpha} b^{\alpha}(b-a)^{\alpha}}{2^{\alpha}}\left[\frac{1}{\Gamma(1+\alpha)} \int_{0}^{1}|1-2 t|^{\alpha p}(d t)^{\alpha}\right]^{\frac{1}{p}}\right. \\
& \left.\quad \int_{0}^{1} \frac{1}{(t b+(1-t) a)^{2 \alpha q}}\left|f^{(\alpha)}\left(\frac{a b}{t b+(1-t) a}\right)\right|^{q}(d t)^{\alpha}\right]^{\frac{1}{q}} .
\end{aligned}
$$


Since $\left|f^{(\alpha)}\right|^{q}$ is generalized harmonically convex on $[a, b]$, thus

$$
\begin{aligned}
\frac{1}{\Gamma(1+\alpha)} & \int_{0}^{1} \frac{1}{(t b+(1-t) a)^{2 \alpha q}}\left|f^{(\alpha)}\left(\frac{a b}{t b+(1-t) a}\right)\right|^{q}(d t)^{\alpha} \\
\leqslant & \frac{1}{\Gamma(1+\alpha)} \int_{0}^{1} \frac{1}{(t b+(1-t) a)^{2 q \alpha}}\left(t^{\alpha}\left|f^{(\alpha)}(a)\right|^{q}+(1-t)^{\alpha}\left|f^{(\alpha)}(b)\right|^{q}\right)(d t)^{\alpha} \\
= & \left(\frac{1}{\Gamma(1+\alpha)} \int_{0}^{1} \frac{t^{\alpha}}{(t b+(1-t) a)^{2 q \alpha}}(d t)^{\alpha}\right)\left|f^{(\alpha)}(a)\right|^{q} \\
& +\left(\frac{1}{\Gamma(1+\alpha)} \int_{0}^{1} \frac{(1-t)^{\alpha}}{(t b+(1-t) a)^{2 q \alpha}}(d t)^{\alpha}\right)\left|f^{(\alpha)}(b)\right|^{q} .
\end{aligned}
$$

Applying the change of the variable $t b+(1-t) a=x$, we have

$$
\begin{aligned}
\frac{1}{\Gamma(1+\alpha)} \int_{0}^{1} \frac{t^{\alpha}}{(t b+(1-t) a)^{2 q \alpha}}(d t)^{\alpha}= & \frac{1}{(b-a)^{2 \alpha}} \frac{1}{\Gamma(1+\alpha)} \int_{a}^{b} \frac{(x-a)^{\alpha}}{x^{2 q \alpha}}(d x)^{\alpha} \\
= & \frac{1}{(b-a)^{2 \alpha}}\left[\frac{1}{\Gamma(1+\alpha)} \int_{a}^{b} \frac{1}{x^{(2 q-1) \alpha}}(d x)^{\alpha}\right. \\
& \left.-\frac{a^{\alpha}}{\Gamma(1+\alpha)} \int_{a}^{b} \frac{1}{x^{2 q \alpha}}(d x)^{\alpha}\right] .
\end{aligned}
$$

Letting $\frac{1}{x^{2 q-2}}=u$ and from $\frac{1}{\chi^{(2 q-1) \alpha}}(d x)^{\alpha}=\frac{1}{(2-2 q)^{\alpha}}(d u)^{\alpha}$, we obtain

$$
\frac{1}{\Gamma(1+\alpha)} \int_{a}^{b} \frac{1}{x^{(2 q-1) \alpha}}(d x)^{\alpha}=\frac{1}{(2-2 q)^{\alpha}} \frac{1}{\Gamma(1+\alpha)} \int_{a^{2-2 q}}^{b^{2-2 q}}(d u)^{\alpha}=\frac{\left(b^{2-2 q}-a^{2-2 q}\right)^{\alpha}}{(2-2 q)^{\alpha} \Gamma(1+\alpha)} .
$$

Letting $\frac{1}{x^{2 q-1}}=u$ and from $\frac{1}{x^{2 q \alpha}}(d x)^{\alpha}=\frac{1}{(1-2 q)^{\alpha}}(d u)^{\alpha}$, we obtain

$$
\frac{1}{\Gamma(1+\alpha)} \int_{a}^{b} \frac{1}{x^{2 q \alpha}}(d x)^{\alpha}=\frac{1}{(1-2 q)^{\alpha}} \frac{1}{\Gamma(1+\alpha)} \int_{a^{1-2 q}}^{b^{1-2 q}}(d u)^{\alpha}=\frac{\left(b^{1-2 q}-a^{1-2 q}\right)^{\alpha}}{(1-2 q)^{\alpha} \Gamma(1+\alpha)} .
$$

Substituting (4.17) and (4.18) into (4.16), we have

$$
\begin{aligned}
\mu_{1}^{\alpha} & =\frac{1}{\Gamma(1+\alpha)} \int_{0}^{1} \frac{t^{\alpha}}{(t b+(1-t) a)^{2 q \alpha}}(d t)^{\alpha} \\
& =\frac{1}{(b-a)^{2 \alpha} \Gamma(1+\alpha)}\left[\frac{\left(b^{2-2 q}-a^{2-2 q}\right)^{\alpha}}{(2-2 q)^{\alpha}}-\frac{a^{\alpha}\left(b^{1-2 q}-a^{1-2 q}\right)^{\alpha}}{(1-2 q)^{\alpha}}\right] .
\end{aligned}
$$

Similarly, we have

$$
\begin{aligned}
\mu_{2}^{\alpha} & =\frac{1}{\Gamma(1+\alpha)} \int_{0}^{1} \frac{(1-t)^{\alpha}}{(t b+(1-t) a)^{2 q \alpha}}(d t)^{\alpha} \\
& =\frac{1}{(b-a)^{2 \alpha}} \frac{1}{\Gamma(1+\alpha)} \int_{a}^{b} \frac{(b-x)^{\alpha}}{x^{2 q \alpha}}(d x)^{\alpha} \\
& =\frac{1}{(b-a)^{2 \alpha} \Gamma(1+\alpha)}\left[\frac{b^{\alpha}\left(b^{1-2 q}-a^{1-2 q}\right)^{\alpha}}{(1-2 q)^{\alpha}}-\frac{\left(b^{2-2 q}-a^{2-2 q}\right)^{\alpha}}{(2-2 q)^{\alpha}}\right] .
\end{aligned}
$$


From Lemma 2.6, by simple calculation, we get

$$
\frac{1}{\Gamma(1+\alpha)} \int_{0}^{1}|1-2 \mathrm{t}|^{\alpha p}(\mathrm{dt})^{\alpha}=\frac{\Gamma(1+\mathrm{p} \alpha)}{\Gamma(1+(\mathrm{p}+1) \alpha)} .
$$

Thus, combining (4.14), (4.15) and (4.19), (4.20), (4.21), we obtain

$$
\begin{aligned}
& \left|\frac{f(a)+f(b)}{2^{\alpha}}-\Gamma(1+\alpha) \frac{a^{\alpha} b^{\alpha}}{(b-a)^{\alpha}} I_{b}^{(\alpha)} \frac{f(x)}{x^{2 \alpha}}\right| \\
& \leqslant \frac{a^{\alpha} b^{\alpha}(b-a)^{\alpha}}{2^{\alpha}}\left[\frac{\Gamma(1+p \alpha)}{\Gamma(1+(p+1) \alpha)}\right]^{\frac{1}{p}}\left[\mu_{1}^{\alpha}\left|f^{(\alpha)}(a)\right|^{q}+\mu_{2}^{\alpha}\left|f^{(\alpha)}(b)\right|^{q}\right]^{\frac{1}{q}} .
\end{aligned}
$$

The proof is completed.

Remark 4.8. In Theorem 4.7, we take $\alpha=1$, which is [7, Theorem 2.7].

\section{Acknowledgment}

This work was supported by the National Natural Science Foundations of China (No. 61672356) and Shaoyang City Science and Technology Plan Project of China(No. 2016GX04). Also, the author is very grateful to editor Prof. Xiao-Jun Yang.

\section{References}

[1] A. Akkurt, M. Z. Sarikaya, H. Budak, H. Yildirim, Generalized Ostrowski type integral inequalities involving generalized moments via local fractional integrals, Rev. R. Acad. Cienc. Exactas Fs. Nat. Ser. A Math. RACSAM, 111 (2017), 797807. 1

[2] M. W. Alomari, M. Darus, U. S. Kirmaci, Some inequalities of Hermite-Hadamard type for s-convex functions, Acta Math. Sci. Ser. B Engl. Ed., 31 (2011), 1643-1652. 1

[3] M. K. Bakula, M. E. Özdemir, J. Pečarić, Hadamard-type inequalities for $\mathrm{m}$-convex and $(\alpha, \mathrm{m})$-convex functions, J. Inequal. Pure Appl. Math., 2008 (2008), 12 pages. 1

[4] G. Chen, H. M. Srivastava, P. Wang, W. Wei, Some further generalizations of Hölder's inequality and related results on fractal space, Abstr. Appl. Anal., 2014 (2014), 7 pages. 2.7

[5] L. Chun, F. Qi, Inequalities of Simpson type for functions whose third derivatives are extended s-convex functions and applications to means, J. Comput. Anal. Appl., 19 (2015), 555-569. 1

[6] S. Erden, M. Z. Sarikaya, Generalized Pompeiu type inequalities for local fractional integrals and its applications, Appl. Math. Comput., 274 (2016), 282-291. 1

[7] İ. İşcan, Hermite-Hadamard type inequalities for harmonically convex functions, Hacet. J. Math. Stat., 43 (2014), $935-942$. $1,1,4.8$

[8] M. A. Latif, M. Shoaib, Hermite-Hadamard type integral inequalities for differentiable m-preinvex and $(\alpha, \mathrm{m})$-preinvex functions, J. Egyptian Math. Soc., 23 (2015), 236-241. 1

[9] H. Mo, X. Sui, Hermite-Hadamard-type inequalities for generalized s-convex functions on real linear fractal set $\mathbb{R}^{\alpha}(0<$ $\alpha<1$ ), Math. Sci., 11 (2017), 241-246. 1

[10] H.-X. Mo, X. Sui, D.-G. Yu, Generalized convex functions on fractal sets and two related inequalities, Abstr. Appl. Anal., 2014 (2014), 7 pages. 1, 2.4

[11] M. E. Özdemir, M. Avci, H. Kavurmaci, Hermite-Hadamard type inequalities via $(\alpha, \mathrm{m})$-convexity, Comput. Math. Appl., 61 (2011), 2614-2620. 1

[12] M. E. Özdemir, Ç. Yıldız, A. O. Akdemir, E. Set, On some inequalities for s-convex functions and applications, J. Inequal. Appl., 2013 (2013), 11 pages.

[13] S. Qaisar, C.-J. He, S. Hussain, A generalizations of Simpsons type inequality for differentiable functions using ( $\alpha, \mathrm{m})$ convex functions and applications, J. Inequal. Appl., 2013 (2013), 13 pages. 1

[14] M. Z. Sarikaya, H. Budak, Generalized Ostrowski type inequalities for local fractional integrals, Proc. Amer. Math. Soc., 145 (2017), 1527-1538. 1

[15] W.-B. Sun, Q. Liu, New Hermite-Hadamard type inequalities for $(\alpha, m)$-convex functions and applications to special means, J. Math. Inequal., 11 (2017), 383-397. 1

[16] W. Sun, Q. Liu, New inequalities of Hermite-Hadamard type for generalized convex functions on fractal sets and its applications, J. Zhejiang Univ. Sci. A, 44 (2017), 47-52. 1 
[17] X.-J. Yang, Local Fractional Functional Analysis and Its Applications, Asian Academic Publisher, Hong Kong, (2011). 2.7

[18] X.-J. Yang, Advanced Local Fractional Calculus and Its Applications, World Science Publisher, New York, (2012). 1, 2, 2.1, 2.2, 2.3, 2.5, 2.6, 2.8, 4.5

[19] Y.-J. Yang, D. Baleanu, X.-J. Yang, Analysis of fractal wave equations by local fractional Fourier series method, Adv. Math. Phys., 2013 (2013), 6 pages. 2

[20] X.-J. Yang, F. Gao, H. M. Srivastava, A new computational approach for solving nonlinear local fractional PDEs, J. Comput. Appl. Math., (in press). 1

[21] X.-J. Yang, F. Gao, H. M. Srivastava, New rheological models within local fractional derivative, Rom. Rep. Phys., 2017 (2017), 12 pages. 1

[22] X.-J. Yang, F. Gao, H. M. Srivastava, Non-differentiable exact solutions for the nonlinear odes defined on fractal sets, Fractals, 2017 (2017), 9 pages. 1

[23] X.-J. Yang, J. T. Machado, C. Cattani, F. Gao, On a fractal LC-electric circuit modeled by local fractional calculus, Comm. Nonlinear Sci. Numer. Simulat., 47 (2017), 200-206. 1

[24] X.-J. Yang, J. A. Tenreiro, D. Baleanu, Exact traveling-wave solution for local fractional Boussinesq equation in fractal domain, Fractals, 2017 (2017), 7 pages. 1 